\title{
Article \\ Virus-Induced Gene Silencing of SIWRKY79 Attenuates Salt Tolerance in Tomato Plants
}

\author{
Yuqing He ${ }^{\dagger}$, Xiaochun Zhang, Yinxiao Tan, Deli Si, Tingting Zhao, Xiangyang Xu, Jingbin Jiang ${ }^{\dagger}$, \\ Huanhuan Yang * and Jingfu Li
}

check for updates

Citation: He, Y.; Zhang, X.; Tan, Y.; Si, D.; Zhao, T.; Xu, X.; Jiang, J.; Yang, H.; Li, J. Virus-Induced Gene Silencing of SIWRKY79 Attenuates Salt Tolerance in Tomato Plants. Agronomy 2021, 11, 1519. https:// doi.org/10.3390/agronomy11081519

Academic Editor: Francis Drummond

Received: 30 May 2021

Accepted: 27 July 2021

Published: 29 July 2021

Publisher's Note: MDPI stays neutral with regard to jurisdictional claims in published maps and institutional affiliations.

Copyright: (c) 2021 by the authors. Licensee MDPI, Basel, Switzerland. This article is an open access article distributed under the terms and conditions of the Creative Commons Attribution (CC BY) license (https:/ / creativecommons.org/licenses/by/ $4.0 /)$.
School of Horticulture and Landscape Architecture, Northeast Agricultural University, Harbin 150030, China; qwe098tgbplm@163.com (Y.H.); z_x_c18639118891@163.com (X.Z.); tyx186970621@163.com (Y.T.); sdlfreedom@163.com (D.S.); ttzhao2016@163.com (T.Z.); xxy709@126.com (X.X.); jjb1248@126.com (J.J.); Lijf_2005@126.com (J.L.)

* Correspondence: huanyaya0126@sina.com

† These authors contributed equally to this work.

Abstract: Previous studies have shown that WRKY transcription factors play important roles in abiotic stress responses. Thus, virus-induced gene silencing (VIGS) was used to identify the function of SlWRKY79 in the salt tolerance of tomato plants by downregulating the expression of the SIWRKY79 gene. Under the same salt treatment conditions, the SIWRKY79-silenced plants showed faster stem wilting and more severe leaf shrinkage than the control plants, and the bending degree of the stem of the SIWRKY79-silenced plants was also greater than that of the control plants. Physiological analyses showed that considerably higher levels of hydrogen peroxide $\left(\mathrm{H}_{2} \mathrm{O}_{2}\right)$, superoxide anion $\left(\mathrm{O}^{2-}\right)$, and abscisic acid (ABA) accumulated in the leaves of the SIWRKY79-silenced plants than in those of the controls after salt treatment. Taken together, our results suggested that SIWRKY79 plays a positive regulatory role in salt tolerance in tomato plants.

Keywords: SIWRKY79; salt resistance; VIGS; tomato

\section{Introduction}

Salt stress is a major abiotic stress that threatens crop production and has significantly affected agriculture [1,2]. In recent years, significantly increased soil salinization levels have seriously affected tomato production [3]. Therefore, it is necessary to identify salt tolerance-related genes, verify their functions, and cultivate salt-tolerant tomato varieties.

The WRKY transcription factor family is one of the largest transcription factor families in plants; these proteins are involved in regulating plant signaling networks [4], and their structures include zinc finger domains [5,6]. The WRKY signaling network responds to both biotic and abiotic stresses [7]. Most studies to date have examined WRKY transcription factors involved in biotic stress, such as disease resistance and immune response mechanisms [8]. However, in recent years, many studies have also reported the roles of WRKY transcription factors in abiotic stress. For instance, microarray analysis in Arabidopsis revealed that 18 AtWRKY genes were involved in salt tolerance regulation [9]. The regulatory function of WRKY 25 was identified by analysis of the WRKY 25 mutant and transgenic Arabidopsis thaliana plants overexpressing WRKY25, and the results showed that WRKY25 played a key role in heat resistance [10]. Transcriptome analysis of rice seedlings under water deficit revealed that several WRKY genes were upregulated under drought conditions, and these genes were involved in the regulation of water deficit stress [11].

Virus-induced gene silencing (VIGS) is an effective method of studying gene function [12,13]; this technique can rapidly decrease gene function by inhibiting gene expression. Transcriptome data revealed that the SIWRKY79 gene is differentially expressed under salt stress, and experiments were performed to silence this gene. The purpose of this study was to identify the function of the SIWRKY79 gene under salt stress. In addition, the 
regulatory relationships of $S I W R K Y 79$ with other genes involved in tomato salt tolerance were detected by quantitative real-time polymerase chain reaction (qRT-PCR) analysis.

\section{Materials and Methods}

\subsection{SlWRKY79 Gene Sequence Analysis}

Two reported genes from Group I, Group II (IIa, IIb, IIc, IId, and IIe), and Group III of the tomato WRKY family were selected, separately. The amino acid sequences were downloaded from the SGN database (https:/ / solgenomics.net/). Sequence alignment was performed with the DNAMAN 8 software, and homology analysis was performed using the MEGA X software.

\subsection{Plant Growth and Treatment}

Tomato (Solanum lycopersicum L. cv. Moneymaker) seedlings were grown in a growth chamber at Dongbei Agricultural University with a 16-h light $\left(24^{\circ} \mathrm{C}\right) / 8$-h dark $\left(20^{\circ} \mathrm{C}\right)$ photoperiod, and the light intensity was 40,000 $1 \times[2,14]$. Eighteen four-leaf stage tomato plants were used. Of these plants, 9 plants were subjected to gene silencing treatment, and 9 plants were used as controls. After the plants were infected with the bacterial solution, the infected plants and the control plants were placed in a light incubator for 3 weeks until photobleaching occurred. At four weeks after treatment, silenced plants and control plants were washed with sterile water, transferred to an Erlenmeyer flask containing 1/2-strength Hoagland nutrient solution for two days, and then transferred to a $200 \mathrm{mM}$ sodium chloride solution for salt stress treatment. The changes in plant phenotype were observed at 0, 4, and $8 \mathrm{~h}$. Samples were taken at each time point for subsequent analysis. Three biological replicates were included.

\subsection{Amplification of the Target Fragment}

The mRNA sequence of the SIWRKY79 gene and Primer 5 software were used to design the following primers containing EcoRI and BamHI digestion sites: 79-F: 5'

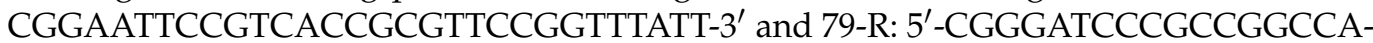
AGGATGGTTATGT- $3^{\prime}$. The primers were analyzed by PCR and agarose gel electrophoresis, and the correct size bands were removed from the gel and purified using a PCR purification kit (Takara) [15]. The purified products were subsequently cloned into a TRV2 vector (Takara) for sequencing.

\subsection{Construction and Infection of the VIGS Vector}

The TRV2 vector and target fragment were double digested with EcoRI and BamHI and then verified by agarose gel electrophoresis. The bands were cut from the gel and recovered using a gel recovery kit. The recovered vector and the target fragment were then ligated with T4 ligase. The ligated vector was transferred into E. coli DH5 $\alpha$ competent cells, and a single colony was selected and grown on liquid Luria-Bertani broth (LB) medium containing $50 \mu \mathrm{g} / \mathrm{mL}$ kanamycin. The cultured bacterial solution was verified by sequencing, and the constructed vector was then extracted with a plasmid extraction kit. TRV1, TRV2-00, TRV2-PDS, and TRV2-SlWRKY79 were transferred into Agrobacterium GV3101 and grown on LB medium containing $50 \mu \mathrm{g} / \mathrm{mL}$ kanamycin and $50 \mu \mathrm{g} / \mathrm{mL}$ rifampicin for $24-48 \mathrm{~h}$. A single colony was selected and incubated in a liquid medium for $24-48 \mathrm{~h}$ until the optical density at $600 \mathrm{~nm}$ wavelength $\left(\mathrm{OD}_{600}\right)=0.5$. Then, the bacteria were centrifuged, and cells were collected and resuspended in IM buffer. The TRV2-00, TRV2-PDS, and TRV2-SIWRKY79 bacterial solutions were mixed individually with the TRV1 bacterial solution at a ratio of 1:1 to obtain the bacterial infection solutions. When the seedlings reached the four-leaf stage, they were used in the virus-induced gene silencing (VIGS) experiment. The bacterial solution was infiltrated into the plant leaves and tender stems with a 1-mL syringe. 


\subsection{Tissue-Specific Expression Analysis}

Roots, stems, and leaves were extracted from the plants at three time points during the stress treatment: 0,4 , and $8 \mathrm{~h}$. The TRIzol method was used to extract RNA from the tomato roots, stems, and leaves, and gene expression levels were analyzed by qRT-PCR as reported in previous studies [15]. EF $\alpha 1$ was used as a reference gene for normalization [15], and the relevant data were analyzed using the $2^{-\Delta \Delta C T}$ method. The TBtools software was used to analyze the expression of the WRKY family genes in tomato tissues and to construct a heat map [16].

\subsection{3,3'-Diaminobenzidine (DAB) and Nitro Blue Tetrazolium e (NBT) Tissue Staining}

The leaves were stained with $\mathrm{DAB}$ and NBT staining solutions to determine the hydrogen peroxide $\left(\mathrm{H}_{2} \mathrm{O}_{2}\right)$ and superoxide anion $\left(\mathrm{O}^{2-}\right)$ contents. $\mathrm{DAB}$ forms a red-brown precipitate when exposed to $\mathrm{H}_{2} \mathrm{O}_{2}$, and $\mathrm{NBT}$ and $\mathrm{O}^{2-}$ react to produce a dark blue precipitate [17].

\subsection{Abscisic Acid (ABA) Assays}

Leaf tissues were homogenized and extracted with $80 \%$ methanol solution. The extraction solution was then collected by centrifugation and extracted again. The extract was filtered, evaporated to increase its concentration, and then adjusted to $\mathrm{pH} 8.0$ with $0.2 \mathrm{~mol} / \mathrm{L} \mathrm{Na}_{2} \mathrm{HPO}_{4}$. The concentrate was extracted twice with a mixture of petroleum ether and ethyl acetate, adjusted to $\mathrm{pH} 2.8$ with $0.2 \mathrm{~mol} / \mathrm{L}$ citric acid, and then extracted three times with ethyl acetate. The extract was condensed and evaporated, dissolved in the mobile phase, and then filtered into a concentration bottle through a membrane.

\section{Results}

\subsection{SlWRKY79 Gene Sequence Analysis}

The protein sequence of SIWRKY79 was compared with those of SIWRKY8, SIWRKY11, SIWRKY17, SlWRKY23, SlWRKY32, SlWRKY33, SlWRKY37, SlWRKY39, SlWRKY45, SlWRKY57, SIWRKY74, SIWRKY80, and SIWRKY81 (Figure 1), and a phylogenetic tree was constructed (Figure 2). SIWRKY79 exhibited the highest homology with SIWRKY37 in the same group.

\begin{tabular}{|c|c|}
\hline 1WRKY8 & KVYSETEICֵ欠VELITYLHFNELVEKTISE SYSEENSRTNS \\
\hline SIWRKY11 & AVKVEVEKYCূSKAITILFTKRKVGKSIGCEAVEVASSTTS \\
\hline SWRKY17 & GNTSTNNKEAГCૂATEATMRKARVSVRARSEAENITLECCW \\
\hline 1WRKY23 & TNAKCূCLIKAKKTVSCૂKKCKKEERFAFNTKSEVLFLELGYRW \\
\hline SIWRKY32 & RRSAPAVNCSTGEVVKCূSSLACৃVIAIVEVIKRETRLGYNW \\
\hline WRKY33 & EVAPICૂSESCূENMSNNEAEVHYCCૂESÇYVRECূKAELGYNW \\
\hline SIWRKY37 & ÇEIASKNRKNACূKVVMTVCૂMEAKSLKCKKNEGEFS. DCWSW \\
\hline SIWRKY39 & LELSSTKKEREEHIKTKTSRVYNRTEFSLTSI IVKLEYCW \\
\hline SIWRKY 45 & FEEASEKRERE . . ITTNVSTVCIKTNRSLCTSVVKLEYNW \\
\hline IWRKY57 & REFSLTASKVKKKEÇKRIRÇEREAFNTKSËVLHLELGYRW \\
\hline SIWRKY74 & KTNRNNELGLLVSÇCূNFTKRARVSVRVRCLAFTMNLECCWW \\
\hline SlWRKY79 & SEKRSRRSIEKRVVSVEIKEIEGSKNKGEISNEESLSWAW \\
\hline SIWRK & GSELKKKKKEKÇLRRGCYKRRKTSGSWNRESATMNLECAW \\
\hline WRK & ELYSSGSCKSSLRRECYKRRKTSESLIKESSLIVLLGHAW \\
\hline
\end{tabular}

Figure 1. Cont. 


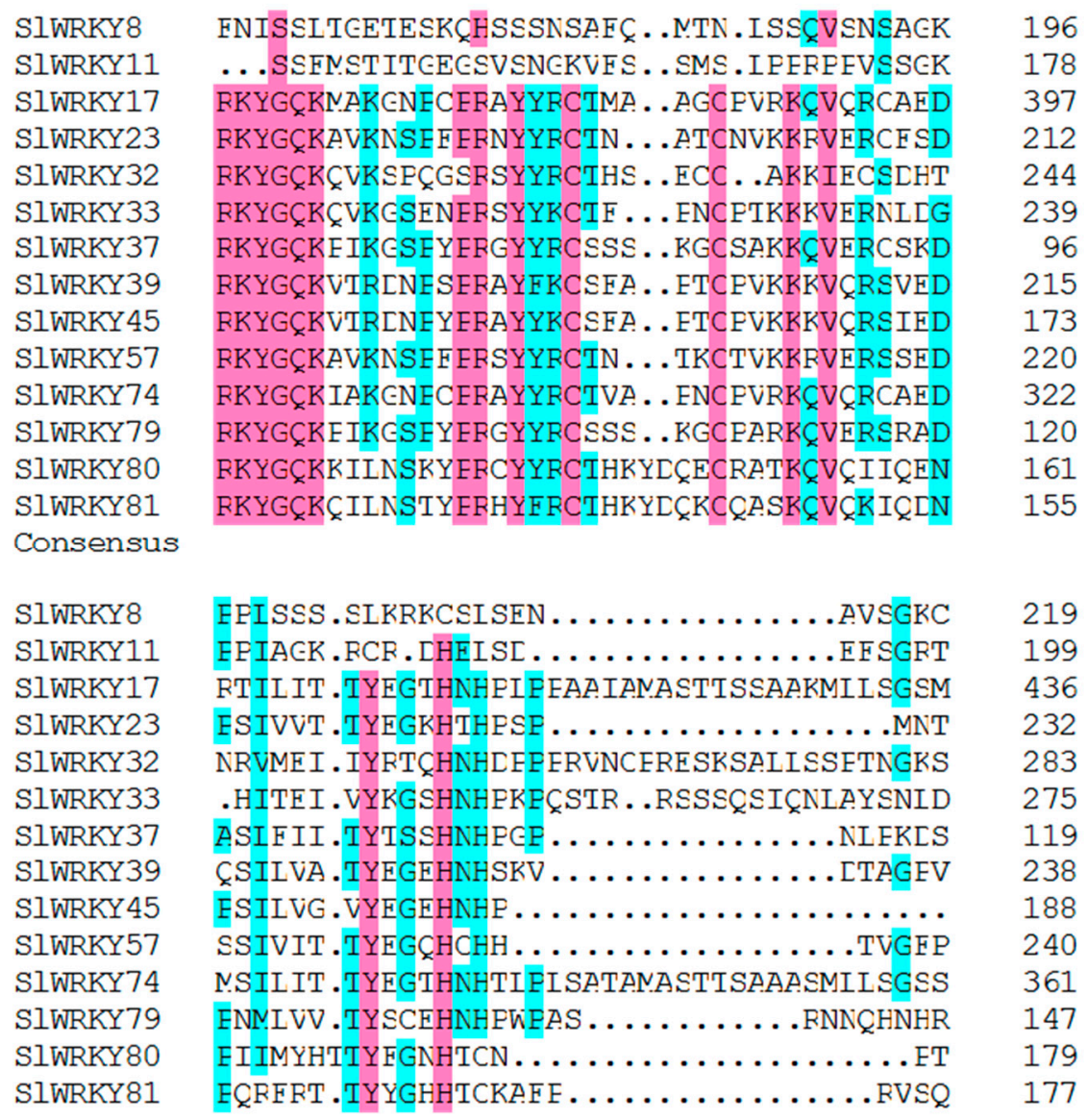

Figure 1. Alignment of the SIWRKY79 protein sequence with those of SIWRKY8, SIWRKY11, SlWRKY17, SIWRKY23, SIWRKY32, SIWRKY33, SIWRKY37, SIWRKY39, SIWRKY45, SIWRKY57, SIWRKY74, SIWRKY80, and SIWRKY81. Pink means higher similarity, light blue means lower similarity.

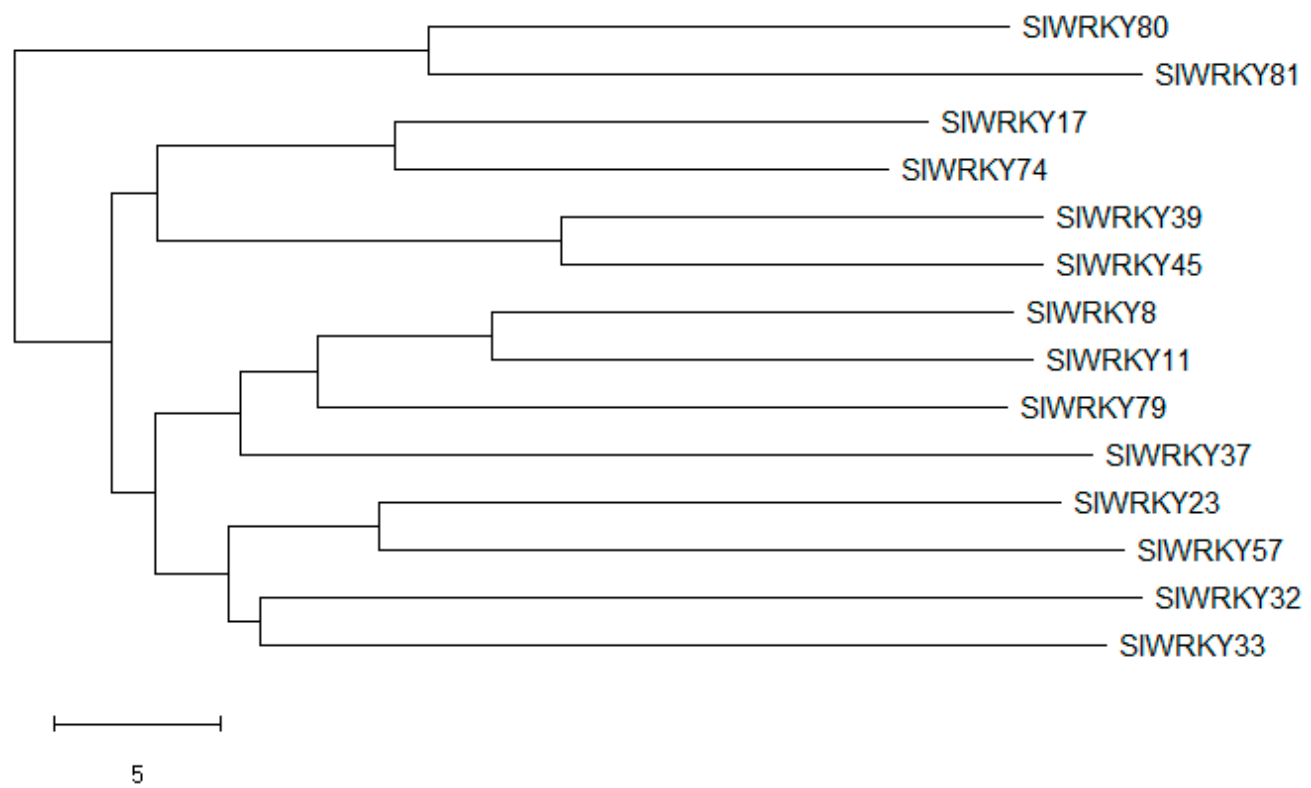

Figure 2. Phylogenetic tree of SIWRKY79, SIWRKY8, SlWRKY11, SIWRKY17, SlWRKY23, SlWRKY32, SlWRKY33, SlWRKY37, SlWRKY39, SIWRKY45, SIWRKY57, SIWRKY74, SIWRKY80, and SIWRKY81. 


\subsection{Plant Phenotype Observation under Salt Stress Treatment}

After $4 \mathrm{~h}$ of salt stress, the TRV2-SlWRKY79 plants showed more wilting than the TRV200 plants. The leaves were slightly curled, and the stems became slightly soft (Figure 3). At $8 \mathrm{~h}$ of salt treatment, the TRV2-SIWRKY79 plants were completely wilted, and the stems had softened completely (Figure 3 ). In addition, the leaves showed severe curling. The TRV2-00 plants had completely wilted at $24 \mathrm{~h}$. In short, the wilting degree of the genesilenced plants was significantly greater than that of the control plants. This phenomenon indicated that the salt tolerance of the plants was reduced after the downregulation of the SIWRKY79 gene.

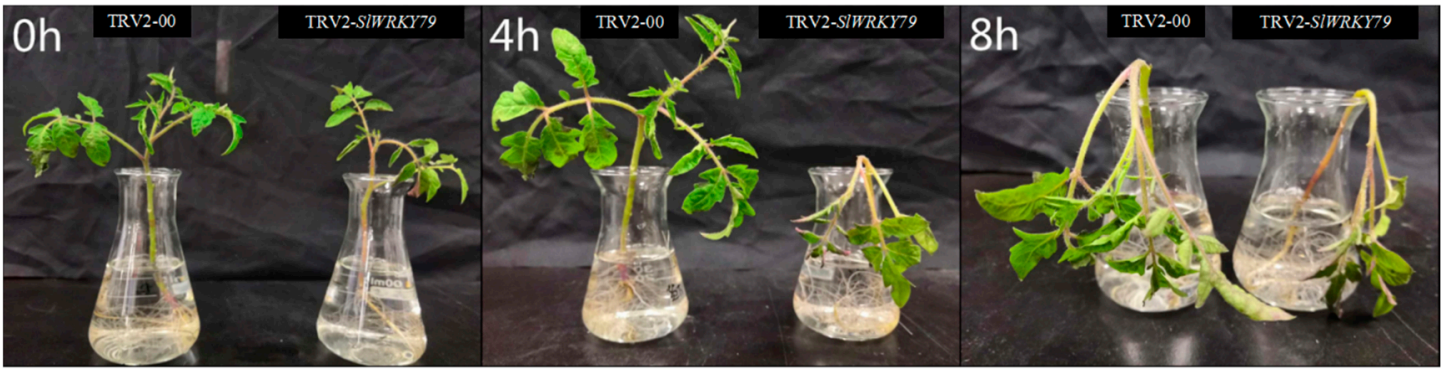

Figure 3. Phenotypic changes in TRV2-00 and TRV2-SlWRKY79 tomato plants during the salt stress treatment.

\subsection{Analysis of SlWRKY79 Gene Expression in Different Tissues after Salt Stress Treatment}

We compared the expression levels of SIWRKY79 in three plant tissues: root, stem, and leaf. No significant differences in SIWRKY79 expression levels of untreated plants were noted during different time periods or in different tissues (Figure 4D). In the treated plants, SIWRKY79 expression in stems at $4 \mathrm{~h}$ was significantly higher than that at $0 \mathrm{~h}$ and $8 \mathrm{~h}$, while SIWRKY79 expression in leaves at $8 \mathrm{~h}$ was significantly higher than that at $0 \mathrm{~h}$ and $4 \mathrm{~h}$ (Figure 4E). In addition, the TRV2-SIWRKY79 plants had lower SIWRKY79 expression levels than the TRV2-00 plants at each time point (Figure 4A-C). Overall, after gene silencing, SIWRKY79 expression levels in the roots decreased, indicating that the SIWRKY79 gene participates in the regulation of salt tolerance in tomato plants.
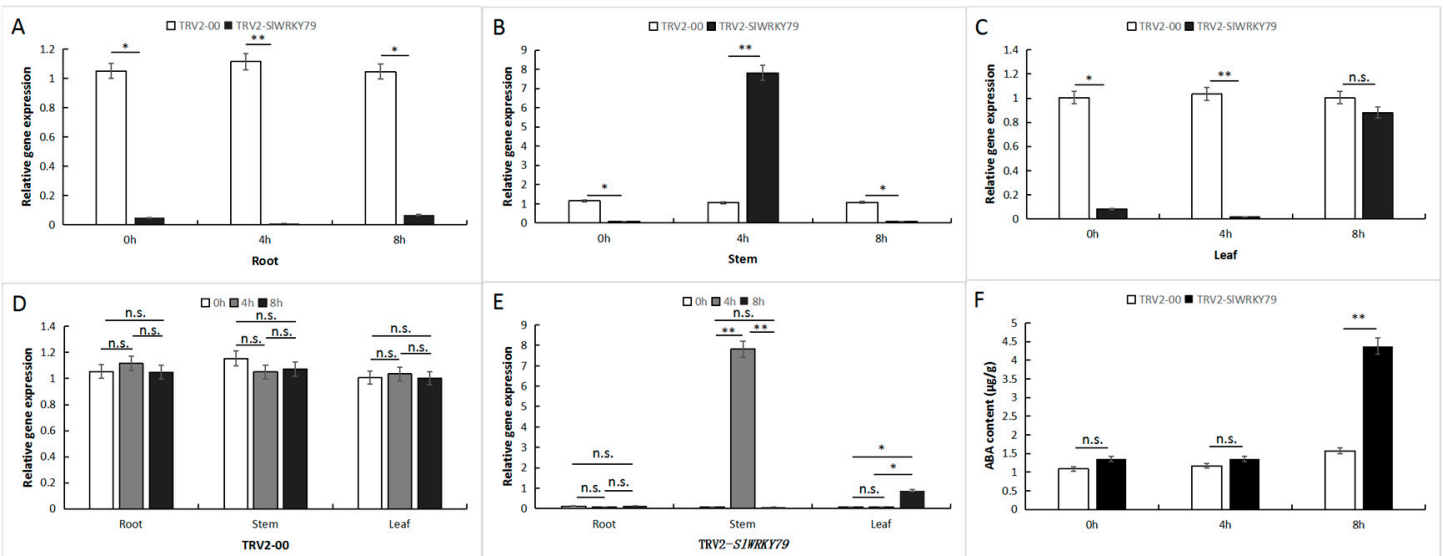

Figure 4. Comparison of SlWRKY79 gene expression levels in roots, stems, and leaves at each time point. (A), Comparison of SIWRKY79 gene expression levels in roots; (B), comparison of SlWRKY79 gene expression levels in stems; (C), comparison of SIWRKY79 gene expression levels in leaves; (D), comparison of SIWRKY79 gene expression levels in the roots, stems, and leaves of TRV2-00 plants at different time points; (E), comparison of SIWRKY79 gene expression levels in the roots, stems and leaves of TRV2-SIWRKY79 plants at different time points; (F), changes in ABA contents in TRV2-00 and TRV2-SlWRKY79 leaves after 0,4 , and $8 \mathrm{~h}$ of treatment. The data presented are the means \pm standard deviations (SDs) of three independent experiments, and the different letters above the columns indicate significant differences at the $p<0.05$ level. ${ }^{*}: 0.01<p<0.05$, **: $p<0.01$, and n.s: $p>0.05$. 
We selected all of the genes of the WRKY transcription factor family and used the TBtools software to analyze the differentially expressed genes in various tissues (Figure 5). The analysis showed that the salt tolerance-related genes SIWRKY13, SIWRKY24, SIWRKY31, SlWRKY50, SlWRKY62, SIWRKY63, and SIWRKY79 were highly expressed in the roots (Figure 5).
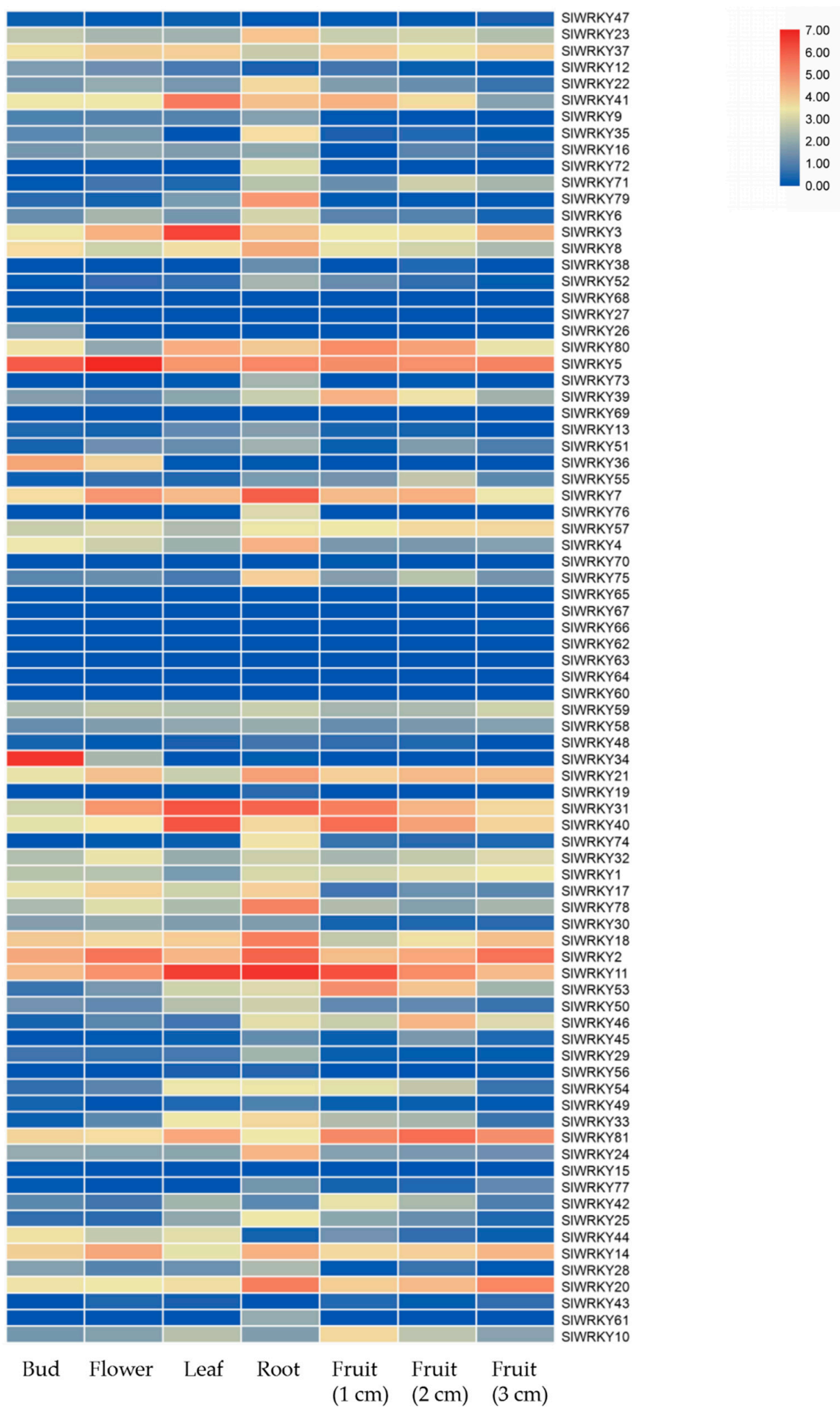

Figure 5. Heat map of differential expression of the WRKY transcription factor family genes in different tissues. 


\subsection{Gene Silencing Significantly Increased Endogenous ABA Content}

During salt treatment, the endogenous hormone ABA gradually accumulated over time. ABA levels in TRV2-SlWRKY79 plants were greater than those of TRV2-00 plants at each time point. At 0,4 and $8 \mathrm{~h}$, the ABA content in TRV2-SIWRKY79 plants was $36 \%, 23 \%$, and $230 \%$ greater than that in TRV2-00 plants, respectively. In addition, the accumulation rate of ABA in the gene-silenced plants was higher, especially from 4-8 h. At this time point, ABA accumulation in the TRV2-SIWRKY79 plants increased by $299 \%$, while that in the TRV2-00 plants increased by only $48 \%$ (Figure 4F). Overall, the ABA level was higher in TRV2-SIWRKY79 plants but only significantly higher at $8 \mathrm{~h}$. These results indicate that the SIWRKY79 gene may negatively regulate the ABA signaling pathway.

\subsection{Salt Treatment Led to Greater ROS Accumulation in TRV2-SlWRKY79 Plants Than in TRV2-00 Plants}

After DAB and NBT staining, the red-brown areas were mainly concentrated at the tip and edge of the leaves (Figure 6A-F), while the dark blue areas were mainly concentrated at the leaf base, in the main vein, and in its vicinity (Figure 6G-L). The stained areas gradually increased with increasing treatment time, and the TRV2-SIWRKY79 plant samples were darker in color than were those of the TRV2-00 plant samples at each time point. These results showed significantly higher ROS accumulation in the TRV2-SIWRKY79 plants compared with the TRV2-00 plants.

$\mathrm{Oh}$

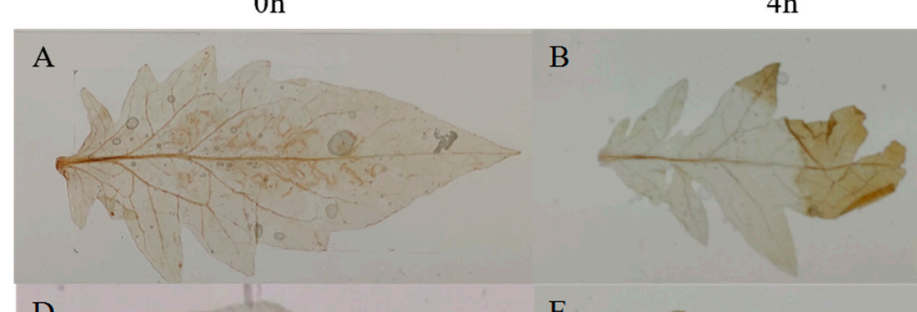

$\mathrm{D}$

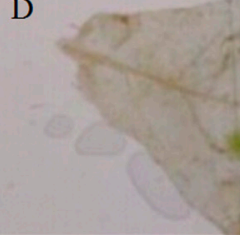

$\mathrm{E}$

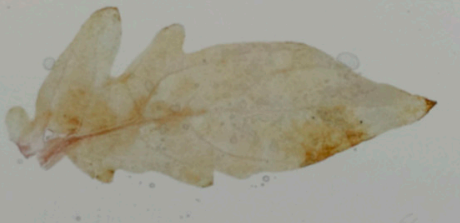

$8 \mathrm{~h}$

$\mathrm{C}$

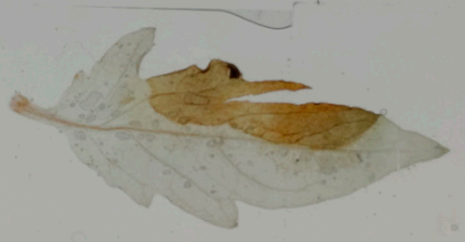

F

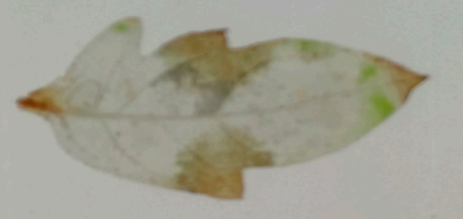

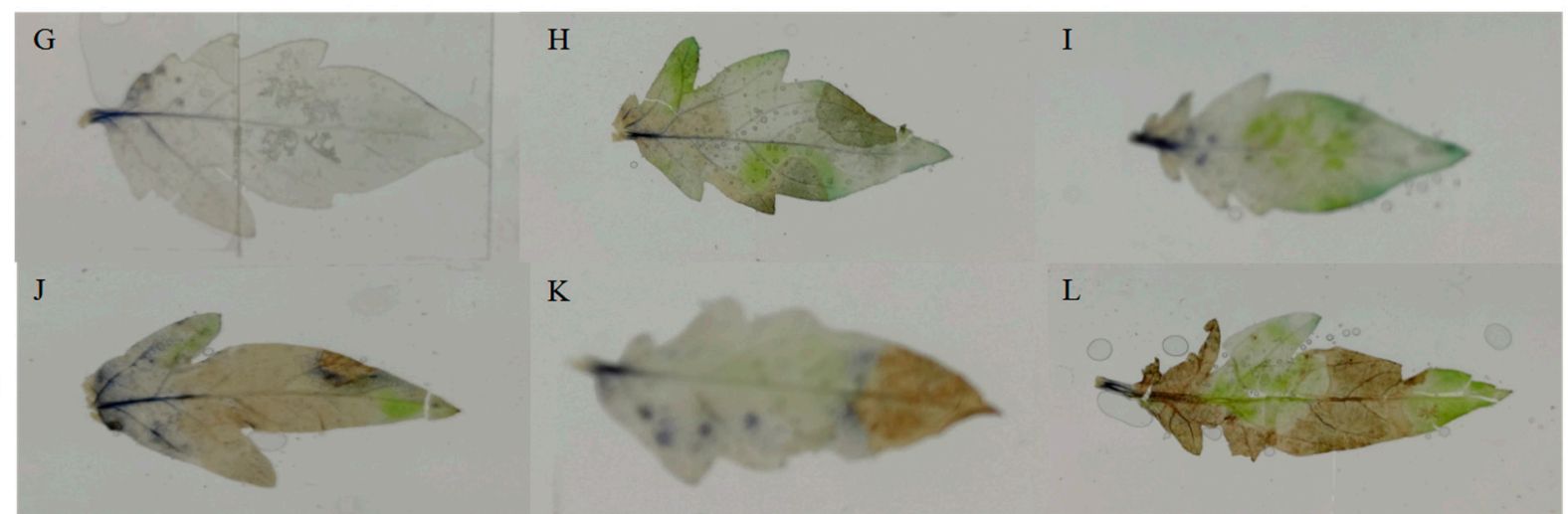

Figure 6. After salt treatment, $\mathrm{H}_{2} \mathrm{O}_{2}$ and $\mathrm{O}^{2-}$ accumulation in leaves increased with increasing time. $(\mathbf{A}-\mathbf{F}), \mathrm{H}_{2} \mathrm{O}_{2}$ accumulation indicated by DAB staining; $(\mathbf{G}-\mathbf{L}), \mathrm{O}^{2-}$ accumulation indicated by NBT staining.

\subsection{SIWRKY79 Gene Silencing Reduced the Expression of Related Salt Tolerance-Related Genes}

The tomato SINAM1, SINAC1, SIWRKY23, and SIRD22 genes were compared, and several salt tolerance-related genes were expressed in plants. The results showed that the expression levels of these genes in TRV2-SIWRKY79 plants were lower than those in 
TRV2-00 plants (Figure 7), and it was hypothesized that SlWRKY79 silencing resulted in a significant decrease in the expression levels of these genes. These results indicate that SINAM1, SINAC1, SIWRKY23, and SIRD22 interact with SIWRKY79 and may be located downstream of the SIWRKY79 gene signaling pathway. Therefore, we proposed a model of SIWRKY79 gene salt tolerance regulation (Figure 8). In this model, external salt stress stimulates SIWRKY79 gene expression and then induces the expression of the downstream SINAM1, SINAC1, SlWRKY23, and SlRD22 genes to resist salt stress.

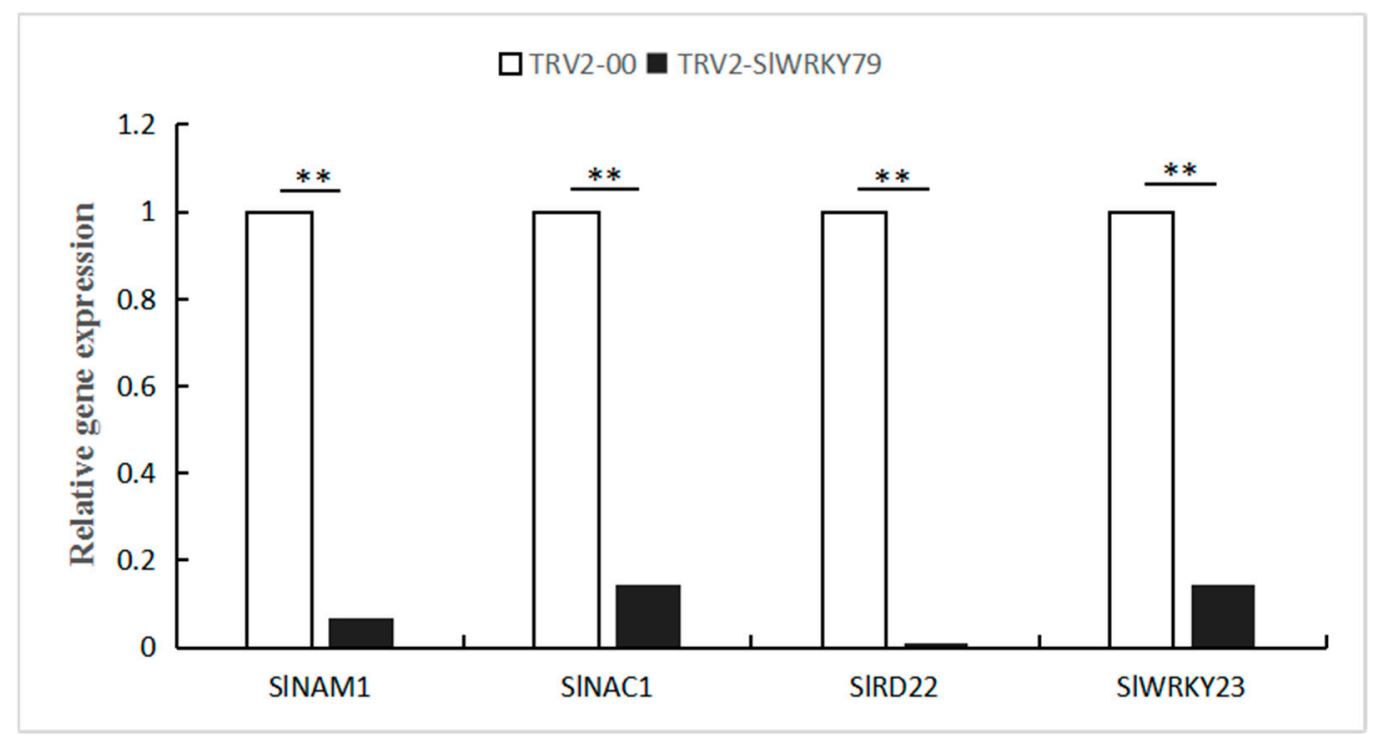

Figure 7. Comparison of SINAM1, SINAC1, SIWRKY23, and SIRD22 expression in TRV2-00 and TRV2-SIWRKY79 cells. The data presented are the means \pm SDs of three independent experiments, and the different symbos above the columns indicate significant differences at the $p<0.05$ level. ${ }^{* *}: p<0.01$.

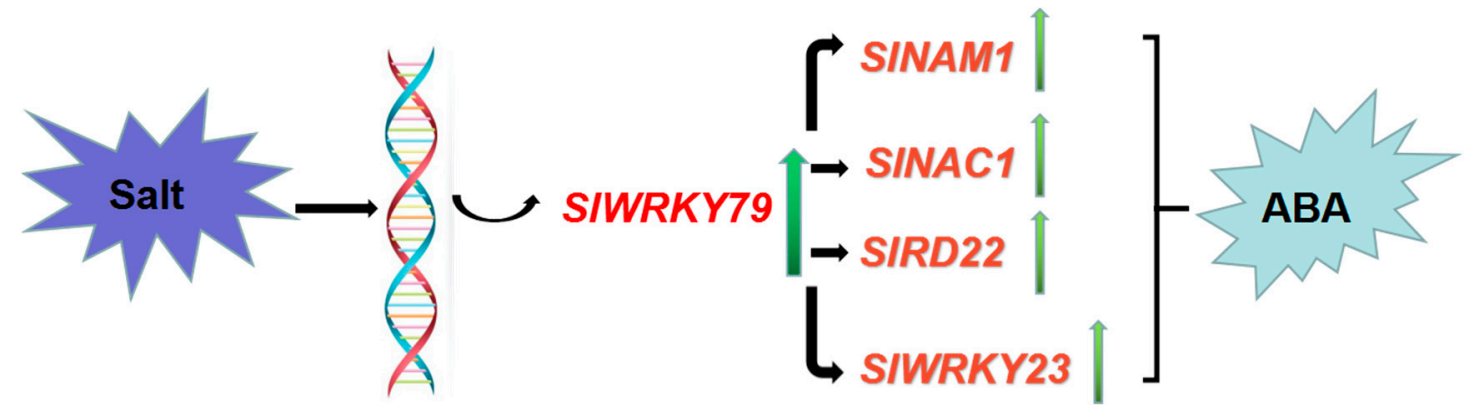

Figure 8. A salt tolerance signaling pathway may be induced by the SIWRKY79 gene.

\section{Discussion}

Plants are subjected to numerous abiotic stresses, such as flooding, low temperature, and salinity, during the growth process, and these stresses can cause great losses in production [18]. Therefore, the study of abiotic stress is particularly important for agriculture. At present, an increasing number of researchers are focusing on functional analyses of WRKY transcription factors in many model plants under abiotic stresses, such as flooding and drought. A series of changes, such as stomatal closure and the expression and induction of related genes, occur in plants in response to abiotic stress [19]. Substantial evidence indicates that WRKY transcription factors participate in [20] and may play very important roles [21] in abiotic stress responses. The SIWRKY79 gene examined in this study belongs to the WRKY family. By silencing the SIWRKY79 gene, we found that it was involved in regulating salt tolerance in tomato plants and had a positive effect on resistance to salt stress. These results are consistent with previous research results and suggest that 
SIWRKY79 participates in stress resistance [15]. However, SIWRKY79 expression levels in the stem of gene-silenced plants was significantly higher than that in control plants at $4 \mathrm{~h}$. We hypothesize that at this time point, the SlWRKY79 gene was still weakly expressed in the stem. This weak expression is a limitation of gene silencing, but the overall gene expression trend decreased, indicating that the SIWRKY79 gene was silenced. The expression of the salt tolerance-related genes SINAM1, SINAC1, SIWRKY23, and SIRD22 in gene-silenced plants was reduced; therefore, we hypothesize that $S I W R K Y 79$ regulates upstream SINAM1, SINAC1, SIWRKY23, and SIRD22 expression and jointly regulates the salt tolerance network mechanism.

ROS are constantly produced in the cells of plants, and their levels are increased in a variety of adverse conditions [17]. These adverse conditions include both biotic and abiotic stresses, such as environmental stress, pathogens, and damage. Under these stress conditions, ROS are produced in large quantities in the photosynthetic apparatus during photorespiration and mitochondrial respiration [22]. This overproduction causes ROS to attack intracellular substances, causing cellular damage $[15,23]$. ROS are associated with many abiotic stresses, as has been shown in Arabidopsis thaliana [18]. In the stress response, ROS are a sign of successful recognition of infection and activation of plant defense, and $\mathrm{O}^{2-}$ and $\mathrm{H}_{2} \mathrm{O}_{2}$ are the most important and stable ROS products [24]. In this study, the ROS content in the plants after gene silencing was apparently greater than that in the unsilenced plants. The $\mathrm{H}_{2} \mathrm{O}_{2}$ produced was mainly concentrated in the leaf edges, while $\mathrm{O}^{2-}$ was mainly concentrated in and around the leaf veins. This ROS overproduction severely damaged the plants, and the plants gradually withered. This phenomenon indicated that the SIWRKY79 gene can positively regulate plant salt stress resistance.

The endogenous hormone ABA is involved in regulating adaptation to environmental stresses [25]. It not only regulates the stomatal opening and the growth and development of plants but also coordinates various stress signal transduction pathways in plants during abiotic stress [26]. Studies have shown that the WRKY family is involved in the ABA signal transduction pathway $[21,27,28]$. In addition, the LTWRKY21 gene, previously isolated from the xerophyte Larrea tridentata (creosote bush), has been shown to activate ABA signaling [29], while WRKY40/WRKY18 and WRKY60 are negative regulators of ABA signaling during seed germination and growth in Arabidopsis thaliana [30]. In this study, the ABA content increased gradually with increasing stress duration, and the amount of ABA accumulation in the SIWRKY79-silenced plants was apparently greater than that in the unsilenced plants. These results indicate that SIWRKY79 is induced by ABA and responds to the ABA signaling pathway. The silencing of this gene significantly reduces the salt tolerance of tomato plants. We discovered for the first time that the SIWRKY79 gene in the WRKY family is involved in salt tolerance in tomatoes. These results were consistent with those of a previous study [31].

Overall, the SIWRKY79 gene was silenced in tomato plants by VIGS, and the SIWRKY79 gene expression response was the most obvious in roots. In addition, SIWRKY79 inhibited ROS and ABA production and improved plant resistance. These results showed that SIWRKY79 may mediate the ABA signaling pathway and salt tolerance in tomato plants. Therefore, we hypothesize that the SIWRKY79 gene plays an active regulatory role in salt tolerance in tomato plants.

Author Contributions: All the authors contributed to the study conception and design. Material preparation, data collection and analysis were performed by Y.H. and J.J. The first draft of the manuscript was written by Y.H. and J.J. and all authors commented on previous versions of the manuscript. All the authors read and approved the final manuscript. Conceptualization, T.Z., X.X. and J.L.; Methodology, Y.H., J.J., X.Z. and H.Y.; Formal analysis, Y.H., J.J., X.Z., Y.T. and D.S.; Writingoriginal draft, Y.H. and J.J.; Writing—review \& editing, Y.H. and J.J.; Supervision, H.Y. All authors have read and agreed to the published version of the manuscript. 
Funding: This work was supported by the Heilongjiang Natural Science Foundation of China (LH2019C037), the National Natural Science Foundation of China. (32002059), the Breeding of high quality and disease resistant new varieties of bulk vegetables. (2019ZX16B02) and the Fellowship of China Postdoctoral Science Foundation (2020 M681068).

Data Availability Statement: Not applicable.

Conflicts of Interest: We declare that we have no conflict of interest.

\section{References}

1. Xiu, Y.; Kirungu, J.N.; Magwanga, R.O.; Xu, Y.; Liu, F. Knockdown of GhIQD31 and GhIQD32 increases drought and salt stress sensitivity in Gossypium hirsutum. Plant Physiol. Biochem. 2019, 144, 166-177.

2. Zhang, X.; Chen, L.; Shi, Q.; Ren, Z. SlMYB102, an R2R3-type MYB gene, confers salt tolerance in transgenic tomato. Plant Sci. 2019, 291, 110356. [CrossRef] [PubMed]

3. Munns, R.; Tester, M. Mechanisms Salinity Tolerance. Annu. Rev. Plant Biol. 2008, 59, 651-681. [CrossRef]

4. Rushton, P.J.; Somssich, I.E.; Ringler, P.; Shen, Q.J. WRKY transcription factors. Trends Plant Sci. 2010, 15, 247-258. [CrossRef]

5. Rushton, P.J.; Macdonald, H.; Huttly, A.K.; Lazarus, C.M.; Hooley, R. Members of a new family of DNA-binding proteins bind to a conserved cis-element in the promoters of alpha-Amy2 genes. Plant Mol. Biol. 1995, 29, 691-702. [CrossRef]

6. Yamasaki, K.; Kigawa, T.; Inoue, M.; Tateno, M.; Yamasaki, T.; Yabuki, T.; Aoki, M.; Seki, E.; Matsuda, T.; Tomo, Y.; et al. Solution Structure of an Arabidopsis WRKY DNA Binding Domain. Plant Cell 2005, 17, 944-956. [CrossRef]

7. $\quad$ Eulgem, T. Dissecting the WRKY Web of Plant Defense Regulators. PLoS Pathog. 2006. [CrossRef]

8. Rushton, P.J.; Torres, J.T.; Parniske, M.; Wernert, P.; Hahlbrock, K.; Somssich, I.E. Interaction of elicitor-induced DNA-binding proteins with elicitor response elements in the promoters of parsley PR1 genes. Embo J. 1996, 15, 5690-5700. [CrossRef] [PubMed]

9. Jiang, Y.; Deyholos, M.K. Comprehensive transcriptional profiling of NaCl-stressed Arabidopsis roots reveals novel classes of responsive genes. BMC Plant Biol. 2006, 6. [CrossRef]

10. Li, S.; Fu, Q.; Huang, W.; Yu, D. Functional analysis of an Arabidopsis transcription factor WRKY25 in heat stress. Plant Cell Rep. 2009, 28, 683-693. [CrossRef]

11. Ray, S.; Dansana, P.K.; Giri, J.; Deveshwar, P.; Arora, R.; Agarwal, P.; Khurana, J.P.; Kapoor, S.; Tyagi, A.K. Modulation of transcription factor and metabolic pathway genes in response to water-deficit stress in rice. Funct. Integr. Genom. 2011, 11, 157-178. [CrossRef] [PubMed]

12. Becker, A.; Lange, M. VIGS-Genomics goes functional. Trends Plant Sci. 2010, 15, 1-4. [CrossRef]

13. Senthil-Kumar, M.; Mysore, K.S. New dimensions for VIGS in plant functional genomics. Trends Plant Sci. 2011, 16, 656-665. [CrossRef]

14. Sun, Y.; Wang, T.; Liu, M.; Nie, Z.; Li, J. Virus-induced gene silencing of SIPKY1 attenuates defense responses against gray leaf spot in tomato. Sci. Hortic. 2020, 264, 109149. [CrossRef]

15. Zhao, T.; Hu, J.; Gao, Y.; Wang, Z.; Bao, Y.; Zhang, X.; Yang, H.; Zhang, D.; Jiang, J.; Zhang, H.; et al. Silencing of the SL-ZH13 Transcription Factor Gene Decreases the Salt Stress Tolerance of Tomato. J. Am. Soc. Hortic. Sci. 2018, 143, 391-396. [CrossRef]

16. Chen, C.; Chen, H.; Zhang, Y.; Thomas, H.R.; Frank, M.H.; He, Y.; Xia, R. TBtools: An integrative toolkit developed for interactive analyses of big biological data. Mol. Plant 2020, 13, 1194-1202. [CrossRef] [PubMed]

17. Kumar, D.; Yusuf, M.A.; Singh, P.; Sardar, M.; Sarin, N.B. Histochemical Detection of Superoxide and H2O2 Accumulation in Brassica juncea seedlings. Bio-Prtocol 2014, 4, 1. [CrossRef]

18. Mittler, R. Abiotic stress, the field environment and stress combination. Trends Plant Sci. 2006, 11, 15-19. [CrossRef] [PubMed]

19. Knight, H.; Knight, M.R. Abiotic stress signalling pathways: Specificity and cross-talk. Trends Plant Sci. 2001, 6, 262-267. [CrossRef]

20. Eulgem, T.; Rushton, P.J.; Robatzek, S.; Somssich, I.E. The WRKY superfamily of plant transcription factors. Trends Plant Sci. 2000, 5, 199-206. [CrossRef]

21. Chen, L.; Song, Y.; Li, S.; Zhang, L.; Zou, C.; Yu, D. The role of WRKY transcription factors in plant abiotic stresses. Biochim. Biophys. Acta 2012, 1819, 120-128. [CrossRef]

22. Mittler, R. Oxidative stress, antioxidants and stress tolerance. Trends Plant Sci. 2002, 7, 405-410. [CrossRef]

23. Andréia, C.; Alice, C.; Patussi, B.S. Antioxidant responses of wheat plants under stress. Genet. Mol. Biol. 2016, 39, 1-6.

24. Torres, M.A. ROS in biotic interactions. Physiol. Plant. 2010, 138, 414-429. [CrossRef]

25. Cutler, S.R.; Rodriguez, P.L.; Finkelstein, R.R.; Abrams, S.R. Abscisic Acid: Emergence of a Core Signaling Network. Annu. Rev. Plant Biol. 2010, 61, 651-679. [CrossRef] [PubMed]

26. Agarwal, P.K.; Jha, B. Transcription factors in plants and ABA dependent and independent abiotic stress signalling. Biol. Plant. 2010, 54, 201-212. [CrossRef]

27. Ren, X.; Chen, Z.; Liu, Y.; Zhang, H.; Zhang, M.; Liu, Q.; Hong, X.; Zhu, J.K.; Gong, Z. ABO3, a WRKY transcription factor, mediates plant responses to abscisic acid and drought tolerance in Arabidopsis. Plant J. Cell Mol. Biol. 2010, 63, 417-429. [CrossRef] [PubMed] 
28. Yan, L.; Liu, Z.Q.; Xu, Y.H.; Lu, K.; Wang, X.F.; Zhang, D.P. Auto- and Cross-repression of Three Arabidopsis WRKY Transcription Factors WRKY18, WRKY40, and WRKY60 Negatively Involved in ABA Signaling. J. Plant Growth Regul. 2013, 32, $399-416$. [CrossRef]

29. Zou, X.; Seemann, J.R.; Neuman, D.; Shen, Q.J. A WRKY Gene from Creosote Bush Encodes an Activator of the Abscisic Acid Signaling Pathway. J. Biol. Chem. 2004, 279, 55770-55779. [CrossRef]

30. Shang, Y.; Yan, L.; Liu, Z.; Cao, Z.; Mei, C.; Xin, Q.; Wu, F.; Wang, X.; Du, S.; Jiang, T.; et al. The Mg-Chelatase H Subunit of Arabidopsis Antagonizes a Group of WRKY Transcription Repressors to Relieve ABA-Responsive Genes of Inhibition. Plant Cell 2010, 22, 1909-1935. [CrossRef]

31. Yan, H.; Jia, H.; Chen, X.; Hao, L.; An, H.; Guo, X. The Cotton WRKY Transcription Factor GhWRKY17 Functions in Drought and Salt Stress in Transgenic Nicotiana benthamiana Through ABA Signaling and the Modulation of Reactive Oxygen Species Production. Plant Cell Physiol. 2014, 55, 2060-2076. [CrossRef] [PubMed] 